\title{
Qui de Léonard de Vinci ou de Marcel Duchamp fait de la Joconde un portrait fallacieux?
}

Wer, Leonardo da Vinci oder Marcel Duchamp, macht aus der Monna Lisa ein trügerisches Porträt?

Who of Leonardo da Vinci or Marcel Duchamp made a false portrait of the Monna Lisa?

\section{Marc Décimo}

\section{CpenEdition}

\section{Journals}

Édition électronique

URL : http://journals.openedition.org/ceg/1599

DOI : $10.4000 /$ ceg. 1599

ISSN : 2605-8359

\section{Éditeur}

Presses Universitaires de Provence

Édition imprimée

Date de publication : 15 juin 2015

Pagination : 229-240

ISBN : 978-2-85399-993-9

ISSN : 0751-4239

\section{Référence électronique}

Marc Décimo, «Qui de Léonard de Vinci ou de Marcel Duchamp fait de la Joconde un portrait

fallacieux? », Cahiers d'Études Germaniques [En ligne], 68 | 2015, mis en ligne le 17 décembre 2017, consulté le 06 décembre 2020. URL : http://journals.openedition.org/ceg/1599 ; DOI : https://doi.org/ 10.4000/ceg.1599

Ce document a été généré automatiquement le 6 décembre 2020

Tous droits réservés 


\section{Qui de Léonard de Vinci ou de Marcel Duchamp fait de la Joconde un portrait fallacieux?}

Wer, Leonardo da Vinci oder Marcel Duchamp, macht aus der Monna Lisa ein trügerisches Porträt?

Who of Leonardo da Vinci or Marcel Duchamp made a false portrait of the Monna Lisa?

Marc Décimo

\section{NOTE DE L'ÉDITEUR}

L'auteur ne souhaite pas la publication électronique de son article.

\section{RÉSUMÉS}

Étant donné L.H.O.O.Q., il s'agit de tenter de comprendre les conditions d'émergence de cette œuvre de Marcel Duchamp qui date de 1919. Pourquoi Duchamp a-t-il ajouté moustaches, barbichette et titre scabreux à cette icône de l'art occidental ? Le seul élan dada explique-t-il cet acte? Que penser de l'interprétation donnée de son œuvre par Duchamp lui-même, quarante ans après, dans l'après-coup de la lecture Freud et du Souvenir d'enfance de Léonard de Vinci? Duchamp a-t-il eu des prédécesseurs? Ne faut-il pas aussi replacer L.H.O.O.Q. dans son contexte pour tenter de l'expliquer au mieux? 
In Anbetracht von Marcel Duchamps L.H.O.O.Q. sollen die Voraussetzungen für das Entstehen dieses Werks im Jahre 1919 untersucht werden. Warum hat Duchamp der Ikone der abendländischen Kunst einen Schnurrbart, einen Spitzbart und einen anstößigen Titel hinzugefügt? Kann allein die dadaistische Geste diesen Akt erklären? Wie können wir Duchamps eigene Werkinterpretation deuten, die er vierzig Jahre später in der Nachträglichkeit seiner Lektüre von Freuds Eine Kindheitserinnerung des Leonardo da Vinci formuliert hatte? Sollte L.H.O.O.Q. nicht auch kontextualisiert werden, um eine dem Werk gerechte Interpretation zu entwickeln?

Given the existence of L.H.O.O.Q., which dates from 1919, we will attempt to understand the conditions of the emergence of this painting. Why did Marcel Duchamp add moustache, goatee and a scabrous title to this icon of Western art? Can this act be merely explained on the basis of a Dada impulse? What are we to think about Duchamp's interpretation of his work 40 years later in the aftermath of his reading Freud and his Leonardo da Vinci and A Memory of His Childhood? Did Duchamp have predecessors? Should we not also place L.H.O.O.Q. in its proper context to endeavour to best explain it?

INDEX

Schlüsselwörter : Duchamp (Marcel), Dada, Monna Lisa, Gender, Diebstahl der Monna Lisa Keywords : Duchamp (Marcel), Dada, Monna Lisa, gender, theft of the Monna Lisa

Mots-clés : Duchamp (Marcel), Dada, Monna Lisa, genre, vol de la Joconde

\section{AUTEUR}

MARC DÉCIMO

Université d'Orléans 